\section{Transient Bilateral Oculomotor Nerve Palsy (TOP) Associated with Ruptured Anterior Communicating Artery Aneurysm: A Case Report}

\section{Abstract}

Oculomotor nerve palsy has been recognized as a localizing sign due to aneurysms along the internal carotid artery, resulting from direct pressure on the nerve. There were only two case reports of an anterior communicating artery (AcomA) aneurysm rupture followed by bilateral oculomotor nerve palsy. We experienced the rare finding of a patient with transient bilateral oculomotor nerve palsy unrelated to consciousness disturbance that started about 38 hours after subarachnoid hemorrhage $(\mathrm{SAH})$ due to AcomA aneurysm rupture. Without raised intracranial pressure, bilateral mydriasis resolved spontaneously 22 hours later. A thick clot Fisher group 3 SAH occupying perimesencephalic, interhemispheric, and sylvian cisterns bilaterally, remained in CT scans after the operation. Reconstructed $\mathrm{MRI}$ data of the oculomotor nerve were converted to a curved multiplanar reconstruction (MPR) image suggested that subarachnoid blood occupying the perimesencephalic cisterns bilaterally induced compression on the oculomotor nerves directly, and the subsequent local edema caused transient bilateral palsy.

Keywords: Oculomotor nerve palsy; Subarachnoid hemorrhage; Aneurysm; Anterior communicating artery; Pupilary dilation

Received: April 11, 2016; Accepted: May 03, 2016; Published: May 10, 2016

\section{TadasukeTominaga ${ }^{1}$, Yasuo Aihara ${ }^{1}$, Takakazu Kawamata ${ }^{1}$ and Koji Arai²}

\section{Tokyo Women's Medical University, Tokyo, Japan \\ 2 Isesaki-Sawa Medical Association Hospital, Japan}

Corresponding author: Yasuo Aihara

झyz3y-aihr@asahi-net.or.jp

M.D. Ph.D., Department of Neurosurgery, Tokyo Women's Medical University, 8-1 Kawada-cho Shinjuku-ku, Tokyo 162-8666, Japan.

Tel: +81-3-3353-8111

Citation: Tominaga T, Aihara Y, Kawamata T, et al. Transient Bilateral Oculomotor Nerve Palsy (TOP) Associated with Ruptured Anterior Communicating Artery Aneurysm: A Case Report. Neurosurg. 2016, 1:2.

\section{Introduction}

Unilateral oculomotor nerve palsy has been recognized as a localizing sign due to aneurysms along the internal carotid artery, particularly at the internal carotid-posterior communicating artery (ICA-PcomA) junction and is considered to be a variable symptom caused by direct pressure on the nerve. Therefore bilateral pupillary dilation in ruptured cerebral aneurysm cases can be misleading in indicating brain herniation or severe global cerebral ischemia.

In this report we describe the rare finding of a patient with transient bilateral oculomotor nerve palsy unrelated to consciousness disturbance that started about 38 hours after subarachnoid hemorrhage due to anterior communicating artery (AcomA) aneurysm rupture. Without raised intracranial pressure, bilateral mydriasis resolved spontaneously 22 hours later. We reviewed and discussed the possible mechanisms of this phenomenon related to an AcomA aneurysm rupture.

\section{Case}

A 67-year-old male was admitted to our hospital because of impaired consciousness. The only previous medical history was of untreated hypertension. On admission, he was deeply comatose and right hemiparesis was evident. Glasgow Coma Scale (GCS) was 8 out of 15 .

A computed tomography (CT) head scan (Figure 1A) was performed and demonstrated a Fisher group 3 subarachnoid hemorrhage (SAH). The CT scan showed subarachnoid blood diffusely occupying perimesencephalic, interhemispheric, and Sylvian cisterns bilaterally. A 3D-CT angiography revealed an anterior communicating artery (AcomA) aneurysm (Figure 1B). No other aneurysms were visualized.

Approximately 8 hours after the onset of coma, the patient underwent an operation of neck clipping and external decompression via anterior inter-hemispheric approach. As subarachnoid blood of interhemispheric cistern was thinner than Sylvian systerns (Figure 1A), we dissected arachnoid 

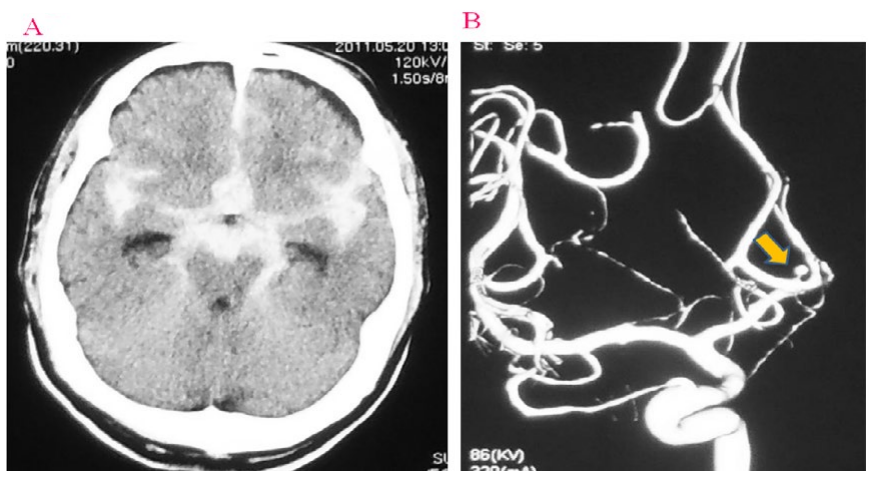

Figure 1A A computed tomography (CT) scan on admisssion demonstrates severe subarachnoid hemorrhage (SAH) occupying both perimesencephalic, interhemispheric, and Sylvian cistern. B: A 3D-CT angiography reveals a saccular aneurysm of the anterior communicating artery (AcomA).

of interhemispheric fissure broadly with ease. An external ventricular drain was placed in the right lateral ventricle during the operation and it remained postoperatively. Shortly before and after the operation, his pupils were equal, reactive, $3 \mathrm{~mm}$ in size. A follow up CT scan showed no significant change.

However, his right pupil suddenly dilated $(5.5 \mathrm{~mm}$ ) approximately 38 hours after the onset ( 27 hours after the operation), without direct or indirect reaction, and the left pupil dilated gradually following the right. About 46 hours after the onset, pupillary size was at the maximum (Figure 2A). Both eyes were abducted, and the findings were considered to be isolated bilateral oculomotor nerve palsy (Figure 2B). During these clinical developments, his consciousness level and clinical condition remained unchanged - GCS 8 (E2VtM5). He moved spontaneously all limbs, and the follow-up CT scan was identical to the post-operative one (Figure 3). Intracranial pressure (ICP) was maintained at $12.5 \mathrm{~mm} \mathrm{Hg}$ by the ventricular drainage postoperatively and later was reduced to $9.5 \mathrm{~mm} \mathrm{Hg}$ for the prevention of brain herniation. Glycerol (200 $\mathrm{ml}$ ) was given i.v. slowly every 12 hours after the operation. In addition, another $200 \mathrm{ml}$ of glycerol was injected immediately after the onset of bilateral pupillary dilation.

After that, his right pupil decreased in size gradually, followed by the left pupil. Direct and indirect reflexes to light returned soon after. Both pupillary sizes were back to normal 22 hours after the onset of the bilateral oculomotor nerve palsies.

A three-dimentional CT (3D-CT) angiography and an MRI study performed approximately 4 weeks after the surgery did not reveal vasospasm sequelae or other abnormalities (Figure 4A and 4B).

\section{MRIData Acquisition and Reconstruction}

In this case, we reconstructed the MRI data to identify the origin and course of the oculomotor nerve. Imaging was performed on a $1.5 \mathrm{~T}$ (Magnetom Avanto, Siemens Healthcare, Erlangen, Germany). The head of the patient was scanned in the supine position and the trajectory of the oculomotor nerve was extracted from each axial MRI images. All MRI row data sets were

\begin{tabular}{|c|c|c|c|c|}
\hline hours after the onset & right pupil size $(\mathrm{mm})$ & left pupil size (mm) & right reflex & left reflex \\
\hline 4 & 3.0 & 3.0 & + & + \\
\hline 12 & 3.0 & 3.0 & + & + \\
\hline 30 & 3.0 & 3.0 & + & + \\
\hline 38 & 5.5 & 3.5 & - & + \\
\hline 40 & 6.5 & 4.0 & - & + \\
\hline 42 & 7.5 & 6.5 & - & - \\
\hline 46 & 8.0 & 7.5 & - & - \\
\hline 52 & 6.0 & 7.5 & + & - \\
\hline 54 & 6.0 & 7.5 & + & - \\
\hline 56 & 5.0 & 7.5 & + & - \\
\hline 58 & 3.0 & 5.0 & + & + \\
\hline 60 & 3.0 & 3.0 & + & + \\
\hline 68 & 2.5 & 2.5 & + & + \\
\hline
\end{tabular}

$\mathrm{B}$

6 hours after the onset 46 hours after the onset

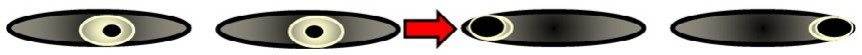

Figure 2A The change of pupillary size and light reflex after the onset (+; reactive, -; not reactive). B : About 46 hours after the onset, both pupils' dilatation was at their maximum and both eyes were abducted as shown on the figure. It indicated bilateral isolated oculomotor nerve palsy.

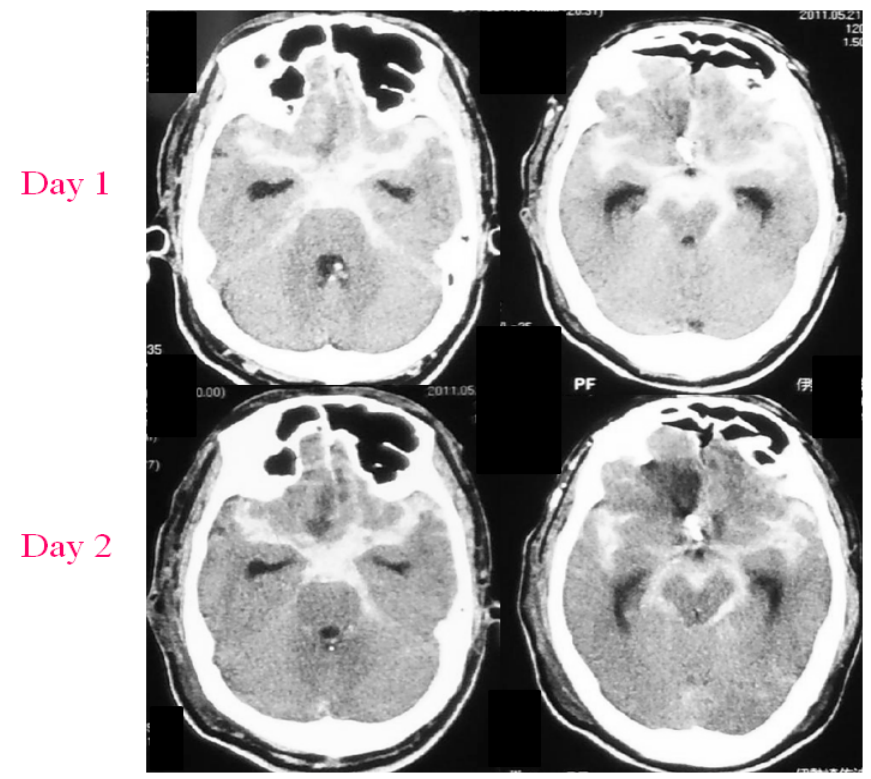

Figure 3 CT scans demonstrating a thick clot remaining around perimesencephalic cysterns and sylvian fissure - one and two days after the operation.

reconstructed to isotropic voxel data using both soft-tissue and bone algorithms [1]. Reconstructed data were transferred to the workstation, and then reconstructed into three-dimensional images (AZE Ltd., Tokyo, Japan). The tract of the right oculomotor nerve is presented on Figure $\mathbf{5 A}$ and $\mathbf{5 B}$. The pathway of the nerve was automatically converted to a curved multiplanar reconstruction (MPR) image as shown in Figure 5C. Thus the long-axis image of the oculomotor nerve appears straight. The right intradural (cisternal and petroclinoid) oculomotor nerve length was $22.7 \mathrm{~mm}$. The left oculomotor nerve was difficult for visualization and reconstruction, and the data were considered unreliable. 

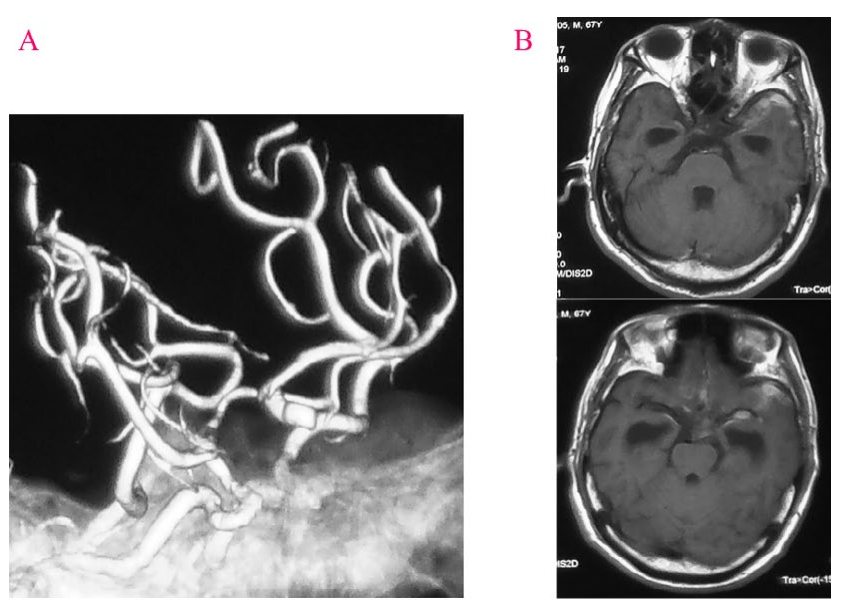

Figure 4 3D-CT angiography (A) and MRI 4 weeks after the operation (B) do not demonstrate any abnormalities. The 3D-CT confirmed the disappearance of the aneurysm.

A

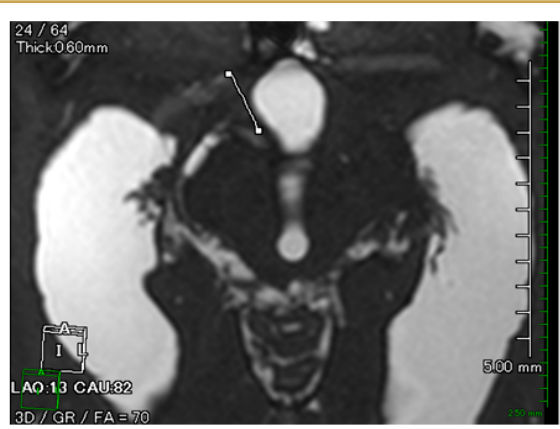

B

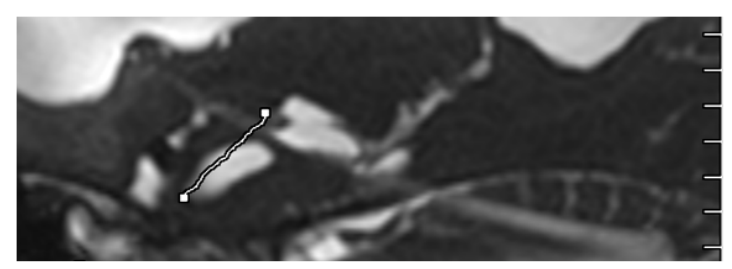

C

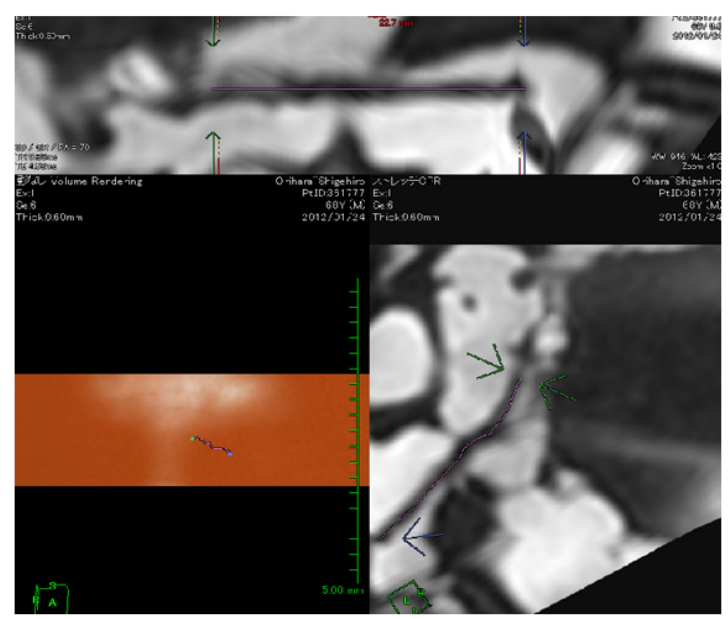

Figure 5 MPR image of head MRI (A:axial view) and (B:sagittal view) which shows the tract of the right oculomotor nerve. C: The pathway of the nerve was automatically converted to a curved multiplanar reconstruction (MPR) image.

\section{Discussion}

Unilateral oculomotor nerve palsy has been recognized as a localizing sign due to aneurysms along the internal carotid artery, particularly at the internal carotid-post communicating artery (ICA-PcomA) junction, resulting from direct pressure on the nerve. Less frequently, aneurysms at the origin of the superior cerebellar artery (BA-SCA) or at the basilar artery (BA) top are associated with these palsies [2-4]. A number of mechanisms of oculomotor nerve palsy in patients with intracranial aneurysms have been classified by Fox [5] as direct or indirect, and peripheral or central. Direct peripheral causes include: 1 ) local pressure by the aneurysm, and 2) hemorrhagic dissection of the nerve. Direct central causes include bleeding into midbrain parenchyma or direct pressure of a large basilar artery on the nucleus. Indirect peripheral causes include increased ICP (from clot, edema, hydrocephalus) causing uncal herniation. Indirect central causes include increased ICP and vasospasm [6]. Each of these mechanisms of oculomotor palsy have different significance as pre- and postoperative signs and the understanding and distinction of them has high clinical significance for complications detection and prognosis.

Suzuki and Iwabuchi reported two cases with unilateral partial oculomotor nerve palsy and bilateral total ophthalmoplegia due to $\mathrm{A} 1$ aneurysm and AcomA aneurysm [7]. They postulated that the oculomotor nerve of a patient with $\mathrm{A} 1$ aneurysm and megadolichobasilar anomaly might be more easily displaced or squeezed between the posterior cerebral and superior cerebellar arteries by increased ICP. However, there were no such anomalies in our case.

There were two similar cases of an AcomA aneurysm rupture followed by bilateral oculomotor nerve palsy, reported by Coyne and Wallace [8], and by Sung Don Kang [9].

In the first case [8], the patient presented with complete right oculomotor nerve palsy 17 hours after SAH symptoms onset, without affected consciousness. Four months after the onset, the bilateral oculomotor nerve palsy recovered. They suggested that persistent ICP elevation without herniation and compression of the oculomotor nerves by focal subarachnoid clot within the perimesencephalic cisterns were the possible mechanism of the palsies.

In the second case [9], the patient presented with sudden bilateral pupillary dilation associated with sudden elevation of ICP to 50 $\mathrm{mm} \mathrm{Hg} 6$ days after onset. However, lateral deviation in the right eye, certainly fixed pupil in the left eye, and both isocoric and reactive pupils remained one year after the event. A follow-up magnetic resonance imaging (MRI) demonstrated infarction of the left medial occipital lobe and lacunar infarction of the pons. They suggested that the direct compression of the nerve derived from the extensive clot in the basal cisterns, uncal herniation, and remote effect due to increased ICP were the mechanisms of nerve injury. Less likely, focal vasospasm might have been the cause of this phenomenon.

Inferences from the clinical course in our case applying the classification of Fox excludes direct pressure from an aneurysm on the third cranial nerves or central causes in the midbrain 
(bleeding, cerebral infarction or large basilar artery). That was confirmed by the postoperative head MRI.

The ICP was maintained constant by the ventricular drainage, and the patient consciousness level was not affected. The postoperative CT head scan and 3D-CT angiography showed no enlargement of subarachnoid blood and no other aneurysm. Therefore increased ICP and uncal herniation could not be a reasonable explanation in this clinical course either.

Cerebral vasospasm, as an indirect central cause, is usually seen in a different time-frame: typical onset at 3 to 5 days after the hemorrhage, a maximum at 5 to 14 days, and a gradual resolution over 2 to 4 weeks $[10,11]$. That last didn't explain the early appearance of the symptoms in our case (2 days after the SAH), and indirect central causes were not an acceptable possibility.

Therefore, we assumed that subarachnoid blood occupying the perimesencephalic cisterns bilaterally induced compression on the oculomotor nerves directly, and the subsequent local edema caused transient bilateral palsy. In both areas there was a "thick" clot on more than 2 CT slices. Therefore, the additional glycerol injection immediately after the onset was effective in reducing the indirect compression.

The fifty-nine human cadaveric dissections by laconetta et al. revealed that the right oculomotor nerve was longer than the left in each segment [12]. The length of the intradural segment of the oculomotor nerve was approximately the same as in our case. However, the presumed length difference between right and left might be related to the time lag of pupil dilation.

\section{Conclusion}

In the rare cases of bilateral oculomotor palsy after SAH in an AcomA aneurysm, thorough diagnostic work-up can indicate it as a "false localizing sign", probably resulting from compression in the perimesencephalic area. Further evaluation is required for more precise understanding of the mechanisms in this condition.

\section{Acknowledgement}

We thank Dr. Kostadin Karagiozov for his advice and manuscript review and David Huang for his guidance and gratefully acknowledge the radiological technologists, nurses, and staff of Departments of Neurosurgery, Tokyo Women's Medical University in preparing this paper.

\section{Declaration of Funding Source}

This study was supported by research funds of Department of Neurosurgery, Tokyo Women's Medical University. 


\section{References}

1 Hasegawa M, Nasuhara Y, Onodera Y, Makita H, Nagai K, et al. (2006) Airflow limitation and airway dimensions in chronic obstructive pulmonary disease. Am J Respir Crit Care Med 173: 1309-1315.

2 Cogan DG, Mount HT (1963) Intracranial Aneurysms Causing Ophthalmoplegia. Arch Ophthalmol 70: 757-771.

3 Laun A, Tonn JC (1988) Cranial nerve lesions following subarachnoid hemorrhage and aneurysm of the circle of Willis. Neurosurg Rev 11: 137-141.

4 Watanabe A, Ishii R, Tanaka R, Tokiguchi S, Ito J (1982) [Relation of cranial nerve involvement to the location of intracranial aneurysms]. Neurol Med Chir (Tokyo) 22: 910-916.

5 Fox JE, Williams B (1984) Central conduction time following surgery for cerebral aneurysm. J Neurol Neurosurg Psychiatry 47: 873-875.

6 Kudo T (1986) Postoperative oculomotor palsy due to vasospasm in a patient with a ruptured internal carotid artery aneurysm: a case report. Neurosurgery 19: 274-277.
7 Suzuki J, Iwabuchi T (1974) Ocular motor disturbances occurring as false localizing signs in ruptured intracranial aneurysms. Acta Neurochir (Wien) 30: 119-128.

8 Coyne TJ, Wallace MC (1994) Bilateral third cranial nerve palsies in association with a ruptured anterior communicating artery aneurysm. Surg Neurol 42: 52-56.

9 Kang SD (2007) Ruptured anterior communicating artery aneurysm causing bilateral oculomotor nerve palsy: a case report. J Korean Med Sci 22: 173-176.

10 Fisher CM, Roberson GH, Ojemann RG (1977) Cerebral vasospasm with ruptured saccular aneurysm-the clinical manifestations. Neurosurgery 1: $245-248$.

11 Heros RC, Zervas NT, Varsos V (1983) Cerebral vasospasm after subarachnoid hemorrhage: an update. Ann Neurol 14: 599-608.

12 laconetta G, de Notaris M, Cavallo LM, Benet A, Enseñat J, et al. (2010) The oculomotor nerve: microanatomical and endoscopic study. Neurosurgery 66: 593-601. 